\title{
Analysis of Artillery Firing Recoil Movement Characteristics Based on Symlet Wavelet Filtering
}

\author{
JIA Chang-zhi, a, HU Zhong-ling ${ }^{1,}$, , XU Dong-sheng ${ }^{1, ~ a, ~ D U A N ~ W e i-r a n ~}{ }^{1, \text { a }}$ \\ ${ }^{1}$ Department of Mechanical Engineering, Ordnance Engineering College, Shijiazhuang, 050003 \\ ${ }^{a}$ E-mail: hzloec@163.com
}

Keywords: symlet wavelet, recoil acceleration, sensor, filtering

\begin{abstract}
Artillery firing recoil movement characteristics concerns artillery firing security and the play of its operational effectiveness, which is the key issue that artillery designers and users focus on. To solve the problems of traditional and modern simulation analysis methods applied to artillery firing recoil movement characteristics, the Symlet wavelet was proposed to analyze recoil movement characteristics. The change law of acceleration, velocity, displacement and other motion parameters were acquired in this method by firstly measuring the artillery recoil acceleration signal when firing, then using Symlet wavelet filtering method to deal with the noise, and finally integrating the signal. Analysis results showed that the Symlet wavelet filtering method can realize the analysis of artillery firing recoil movement characteristics, which can provide references for the design and use of artillery.
\end{abstract}

\section{Introduction}

In order to ensure the safety and effectiveness of artillery firing, artillery firing recoil movement characteristics require to be analyzed in the process of artillery design and operation. Traditional analysis methods were to compute positive and negative issues of artillery recoil. The calculation of positive and negative issues of artillery recoil was achieved mainly by establishing the artillery recoil movement differential equations, analyzing the stress of artillery parts, solving the artillery recoil mechanism structure parameters, or using all the structures of the recoil mechanism, and solving actual recoil resistance and movement parameters, to analyze artillery firing recoil movement characteristics [1]. Because the differential equation of recoil issue was established on the basis of multiple hypotheses, its calculation results would have certain error inevitably. And with the development of the computer technologies, the simulation technology has been widely used to build the virtual prototype model of artillery to analyze artillery firing recoil movement characteristics when artillery is firing. However, the simulation model must be validated by the full test data to ensure its reliability. Therefore, it is necessary to research the methods of artillery recoil movement characteristics test, especially for the artillery that has been finalized or largely equipped. As sensor technology is developing fast in the technology and market condition, fully considering the actual test method for analysis of artillery firing recoil movement characteristics has been possible. Based on a type of artillery as study object, in this paper, Symlet wavelet was used to analyze artillery firing recoil movement characteristics, by measuring the artillery recoil acceleration when it is firing. Symlet wavelet filtering method was used to deal with the noise. And then on the basis of test data, by step-by-step integration method, artillery recoil velocity and recoil displacement and other recoil movement characteristics Wwere analyzed.

\section{Symlet wavelet}

Wavelet transform is a new mathematical tool rises in the field of signal analysis in recent years and has been widely used. Its basic principle is analyzing the data at the same time in the different frequency scale decomposition results of all the data available features so as to realize signal analysis [2]. Lke the signal decomposed into a series of Fourier analysis of superposition of sine waves with different frequencies, the signal is decomposed into a series of wavelet analysis, which 
is a superposition of wavelet function, and the wavelet function is controlled by a wavelet base through translation and scale expansion [3]. With irregular wavelet function approximation method makes the signals are analyzed with wavelet in time domain has the ability of the excellent local analysis information, thereby non-stationary signal can be analyzed effectively. It also shows the superiority of wavelet approximation signal is determined by the wavelet base. In wavelet analysis, therefore, a suitable wavelet base should be chosen, from more than ten wavelet bases which are used commonly, by its characteristics such as compactly supported characteristic, attenuation, regularity and symmetry. In this paper, the actual characteristics of artillery recoil acceleration signal was combined, so Symlet wavelet was chosen to achieve artillery recoil acceleration signal processing.

Symlet wavelet was found by French scholar Ingrid Daubechies by improving the structure on the basis of $\mathrm{dbN}$ wavelet. Same as the $\mathrm{dbN}$ wavelet, Symlet wavelet has orthonormal base of compactly supported. The effective support length of its scale function is $2 N-1$, disappear moment is $\mathrm{N}$, and there is no fixed expressions or wavelet base. But its discrete wavelet transform modulus square has a fixed expression.

When the discrete form of the wavelet is $m_{0}(\omega)=\frac{1}{\sqrt{2}} \sum_{k=0}^{2 N-1} h_{k} e^{-i k \omega}$, its modulus square is [4]

$$
\left|m_{0}(\omega)\right|^{2}=\left[\cos ^{2}(\omega / 2)\right]^{N} P\left[\sin ^{2}(\omega / 2)\right]
$$

Where, $P(y)=\sum_{k=0}^{2 N-1} C_{k}^{N-1+k} y^{k}$

$\omega$ is the angular vector; $h_{k}$ is a parameter.

But Symlet wavelet has approximate symmetry, while the dbN wavelet has no symmetry except $N=1$. Because symmetric and antisymmetric wavelet function and scaling function can be constructed out of compactly supported regular wavelet base, and have a linear phase, in signal analyses, scale function and wavelet function can be used as a filter. A filter with a linear phase can avoid the signal distortion during the wavelet decomposition and reconstruction. Therefore, this article adopted the method of Symlet wavelet filter to deal with the noise of artillery recoil acceleration signal, and effectively avoided recoil acceleration signal distortion during decomposition and reconstruction.

\section{Artillery recoil acceleration signal processing based on Symlet wavelet}

\section{A. Test of artillery recoil acceleration}

When the gun is shooting, the recoil motion is accompanied by a strong impact vibration, which causes the recoil acceleration signal contains all kinds of high frequency and low frequency signals, so the sensor tests recoil acceleration must have a large measuring range and good frequency response. In this article, a YD-PC piezoelectric acceleration sensor with sampling frequency of $5 \mathrm{kHz}$ was chosen to test artillery recoil acceleration. In order to obtain high frequency response, sensor installation, adopts bolt connection method, the sensor is fixed by bolts left after the tail of the gun. This connection mode allows the sensor to collect signal under the high frequency response, and at the same time ensures that the entire signal acquisition process will not affect the operation of using personnel. The sensor installation is shown in Figure 1. 


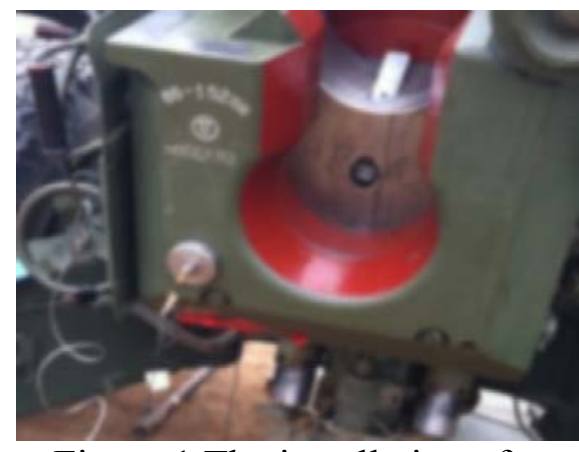

Figure 1 The installation of sensor

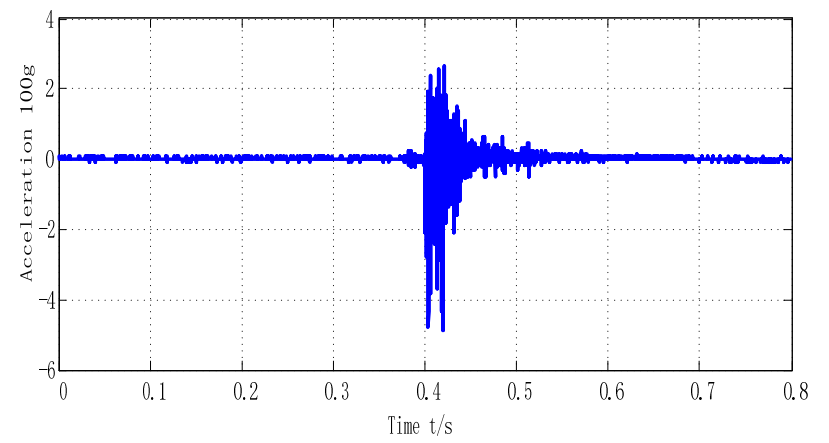

Figure 2 The signal of artillery recoil acceleration

The signal from sensor testing input to the oscilloscope through the charge amplifier, and the acceleration signal was collected by a computer software acquisition terminal at the same time. According to the characteristics of artillery recoil motion, the signal was collected within $0.8 \mathrm{~s}$ since the gun was shooting. The signal of artillery recoil acceleration by acquisition is illustrated in Figure 2.

This signal was collected by firing test under the condition of $2 \#$ charging, $11.3^{\circ} \mathrm{C}$ powder charge temperature. Test results showed that the sensor and signal acquisition device can effectively capture artillery recoil acceleration. And preliminary analysis according to the characteristics of the acceleration signal could be obtained. Artillery recoil time was no more than $0.3 \mathrm{~s}$, also the movement of recoil part is accompanied by a large number of high-frequency impact vibration, cannot reflected artillery recoil movement characteristics intuitionisticly, further filtering de-noising processing was acquired.

\section{B. Symlet wavelet based recoil acceleration signal processing}

The essence of processing the recoil acceleration signal based on Symlet wavelet is to look for the best mapping from actual artillery recoil acceleration signal space to Symlet wavelet function space, which can recover original signal on the greatest degree. The basic flow of the de-noising processing is shown in Figure 3.

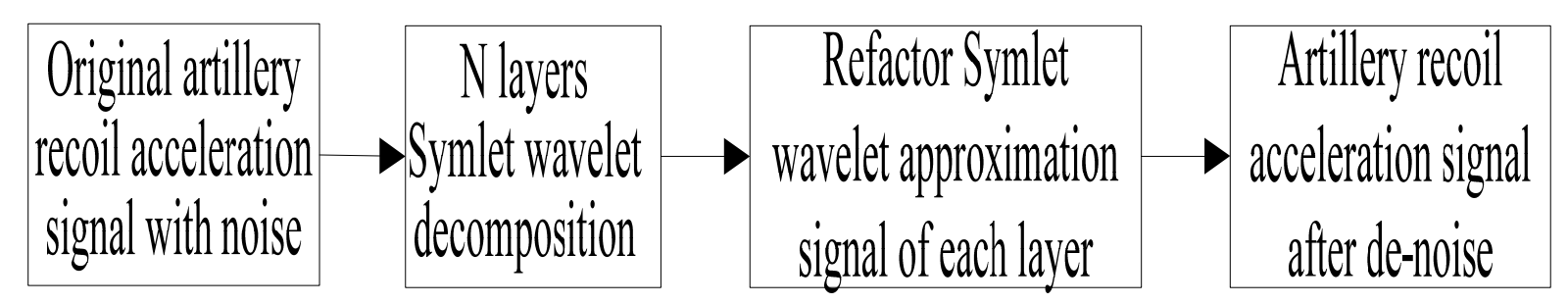

Figure 3 The basic flow of the de-noising processing

Using Symlet wavelet to achieve wavelet decomposition of artillery recoil acceleration signal, the selection of wavelet basis and decomposition layers is mainly considered. Through simulation comparison, selecting Sym3 wavelet as the wavelet base was more appropriate. And in theory, for Symlet wavelet decomposition, the higher layer number is used, the better results can be got. But this will greatly increase the complexity of operation. Simulation results indicate that the wavelet approximation signal in each layer has highlighted the artillery launch recoil acceleration change law if no less than 5 layers decomposition levels are chosen, therefore 5 layers are chosen in this paper as decompose layers. For the artillery recoil acceleration signal, Sym3 wavelet is choosed as the wavelet base, with 5 layers of decomposition, refactor again, the detail coefficients of each layer are shown in Figure 4, the refactoring approximation signal of each layer is shown in Figure 5. 


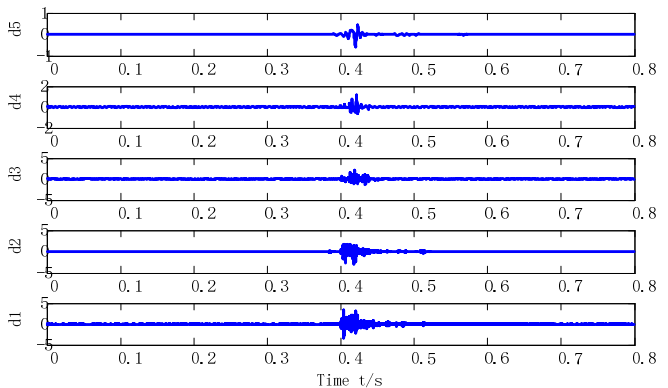

Figure 4 The detail coefficients of each layer Figure 5 The refactoring approximation signals of each layer

It can be found through Symlet wavelet decomposition, for the movement of recoil part when artillery is firing, that the recoil energy mainly concentrates in the 5th layer approximation signal (a5), as shown in Figure 6; and compound into energy mainly concentrates in the 4th layer of the details (d4), as shown in Figure 7.

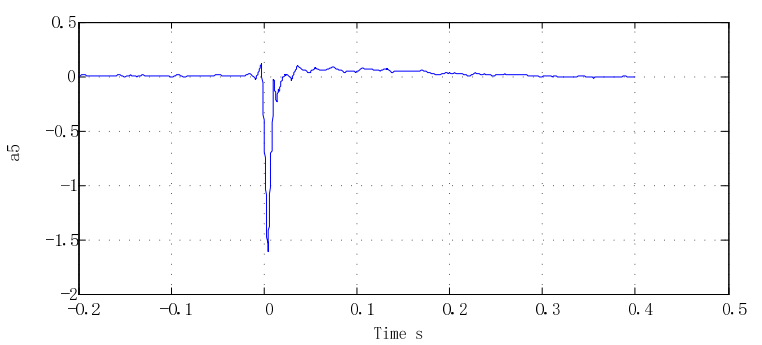

Figure 6 The 5th layer approximation signal

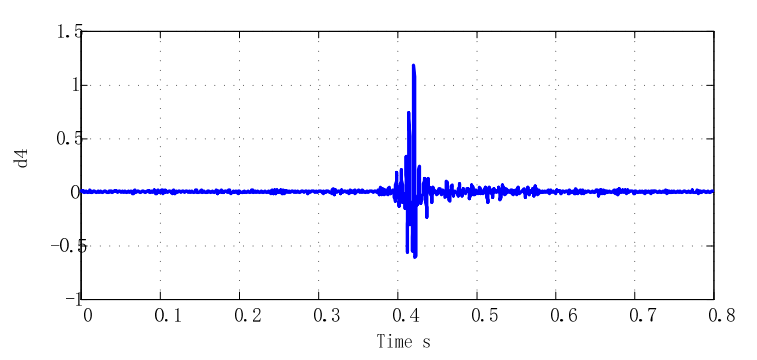

Figure 7 The 4th layer of the details

\section{Artillery firing recoil movement characteristics analysis}

According to Symlet wavelet analysis of recoil acceleration, artillery recoil energy mainly concentrates in the 5th layer. Therefore the 5th layer approximation signal to analyze the movement characteristics of recoil part when artillery is firing was studied. As shown in Figure 8, the curve of artillery firing recoil velocity can be obtained from the integral of the 5th layer approximation signal of Symlet wavelet, moreover the curve also shows that artillery recoil time is about $0.2 \mathrm{~s}$ and the largest artillery recoil velocity is about $10 \mathrm{~m} / \mathrm{s}$.

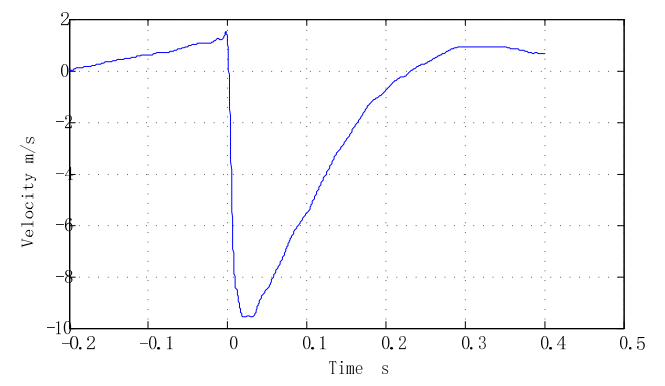

Figure 8 The curve of artillery recoil velocity

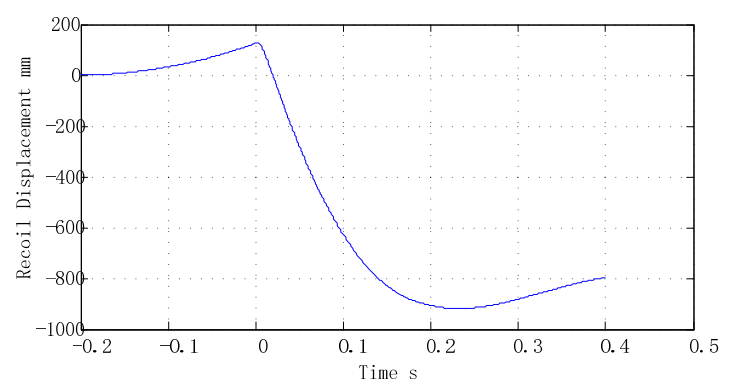

Figure 9 The curve of artillery recoil displacement

The curve of artillery recoil displacement changing over time can be obtained through further integral of the artillery recoil velocity, which is shown in Figure 9. The curve also shows the artillery recoil time is about $0.2 \mathrm{~s}$, the largest recoil displacement is $918 \mathrm{~mm}$, and the actual artillery recoil length is $926 \mathrm{~mm}$ in this experiment. It shows that the recoil acceleration signal can get de-noising processing effectively by Symlet wavelet analysis, and the artillery recoil velocity curve and recoil displacement curve can be obtained, by integration of each layer of fully contains recoil energy approximation signal, to realize the analysis of artillery recoil movement characteristics. 


\section{Conclusions}

When artillery is firing, its movement characteristics reflect the state of the whole recoil parts. Analyzing it can effectively ensure the safety of artillery firing and the play of combat effectiveness, disadvantages exist in the use of traditional recoil issue calculation and the analysis of the modern simulation modeling method for the movement characteristics. In this paper, an analysis method of artillery firing recoil movement characteristics was proposed based on Symlet wavelet filtering to complete de-noising of artillery recoil acceleration signal from test and to obtain the hierarchical approximation signal which reflects the major part of the recoil energy, and the change law of artillery firing recoil velocity and displacement were gained via further integral calculations. Results show that using Symlet wavelet can effectively eliminate the noise in artillery recoil acceleration test signal, and the change rule of artillery firing recoil velocity and displacement can be obtained, so the analyses of artillery firing movement characteristics can be realized.

\section{References}

[1] Du Zhong-hua, Han guo-zhu, Wang rui-lin. Cannon Design Principle [M]. Shijiazhuang: Ordnance Engineering College, 2007.

[2] Fang Shu-guang. The Application of Wavelet Based on Time-frequency Analysis Method in Ultrasonic Signal Processing [D]. Shandong University, 2009.

[3] Ge Zhe-xue, Sha Wei. Wavelet Analysis Theory and MATLABR2007 Implementation [M]. Electronic Industry Press, 2007

[4] Zhu Jian-bing, Ji You-liang, Zhao Pei-kun, etc. Application of wavelet transform in auto-identify units of stratigraphy sequence [J]. Petroleum Exploration and Development, 2005, 32 (1) : 84-86 